\title{
A Neonate Diagnosed with Wolff-Parkinson-White Syndrome Presenting with Cardiogenic Shock
}

\author{
Ji Eun Ha, $\mathrm{MD}^{1}$, Sun Hyang Lee, $\mathrm{MD}^{1}$, Ga Young Park, $\mathrm{MD}$, $\mathrm{PhD}^{1}$, Young-Lim Shin, $\mathrm{MD}, \mathrm{PhD}^{1}$, Sung Shin Kim, $\mathrm{MD}$, \\ $\mathrm{PhD}^{1}$, and Mi-Ae Jang, $\mathrm{MD}, \mathrm{PhD}^{2}$ \\ Departments of ${ }^{1}$ Pediatrics and ${ }^{2}$ Laboratory Medicine and Genetics, Soonchunhyang University Bucheon Hospital, Soonchunhyang \\ University College of Medicine, Bucheon, Korea
}

\section{ABSTRACT}

We present the case of a healthy 28-day-old female full-term neonate who was admitted to the neonatal intensive care unit for severe metabolic acidosis, hypoglycemia, and an initial sinus rhythm. The first diagnostic hypothesis was hypovolemic shock, and fluid resuscitation was started immediately. During fluid therapy, cardiovascular collapse occurred with supraventricular tachycardia. The latter was successfully treated with adenosine and beta-blockers. After 8 days, electrocardiography showed ventricular pre-excitation, and Wolff-Parkinson-White syndrome was diagnosed. A novel variant of the $M Y L 2$ gene that is related to hypertrophic cardiomyopathy and conduction defect was found after discharge. Cardiogenic shock should be considered, despite being a rare cause of shock in neonates.

Key Words: Shock, cardiogenic; Cardiomyopathy, hypertrophic; Acidosis; Tachycardia, supraventricular; Wolff-Parkinson-White syndrome

\section{INTRODUCTION}

Wolff-Parkinson-White (WPW) syndrome is rare among neonates; however, the first manifestation may be shock, which requires immediate attention. When supraventricular tachycardia (SVT) occurs in neonates, symptoms of acute heart failure may appear, manifesting as reduced oral intake, lethargy, and breathing difficulty. If cardiovascular collapse is sustained for a long time, severe metabolic acidosis may occur. We report the case of a healthy 28-day-old female full-term neonate diagnosed with WPW syndrome after cardiogenic shock with non-specific symptoms and SVT. This case report alerts pediatricians to exercise a high index of suspicion for arrhythmia, which is a critical cause of cardiogenic shock, and emphasizes the importance of evaluating suspected cases with serial electrocardiography (ECG).
Received: 13 January 2021

Revised: 26 March 2021

Accepted: 29 March 2021

Correspondence to: Sun Hyang Lee, MD

Department of Pediatrics, Soonchunhyang University Bucheon Hospital, Soonchunhyang University College of Medicine, 170 Jomaru-ro, Wonmi-gu, Bucheon 14584, Korea

Tel: +82-32-621-5420

Fax: +82-32-621-5018

E-mail: hdsunhyang80@hanmail.net

\footnotetext{
Copyright(c)

By Korean Society of Neonatology. All right reserved.

This is an Open-Access article distributed under the terms of the Creative Commons At tribution Non-Commercial License (http:// creativecommons.org/licenses/by-nc/4.0), which permits unrestricted non-commercial use, distribution, and reproduction in any medium, provided the original work is pro perly cited.
} 


\section{CASE REPORT}

A previously healthy 28-day-old female was brought to the emergency room due to poor feeding. She was born at 39 weeks' gestation via spontaneous vaginal delivery with a birth weight of $3,020 \mathrm{~g}$. She had visited the local clinic 3 days earlier. Echocardiography was performed at the local clinic to evaluate a gallop rhythm and cardiac murmur, but no abnormalities were found. However, her poor feeding and lethargy worsened the day before she visited our hospital. She did not present with any other signs. Her vital signs were as follows: heart rate (HR) of 160 beats per minute (bpm), respiratory rate (RR) of 30 breaths/min, oxygen saturation of $98 \%$ on room air, and body temperature (BT) of 36.6 ${ }^{\circ} \mathrm{C}$. Initial laboratory tests showed hypoglycemia (serum glucose, $42 \mathrm{mg} / \mathrm{dL}$ ) and metabolic acidosis (venous blood gas analysis [VBGA]: $\mathrm{pH}, 7.41 ; \mathrm{PCO}_{2}, 24 \mathrm{kPa}$; bicarbonate, $15.5 \mathrm{mmol} / \mathrm{L}$; and base excess, $-6.5 \mathrm{mmol} / \mathrm{L})$. Initial chest radiograph showed mild cardiomegaly without pulmonary edema (Figure 1). To correct this, a bolus of dextrose was administered and fluid resuscitation was started. Despite these measures, she became lethargic and showed cyanosis 5 hours later and developed hypothermia and tachycardia (HR, 220 bpm; blood pressure, 93/71 mm Hg; RR, 50 breaths/min; oxygen saturation, $78 \%$; and $\mathrm{BT} 33^{\circ} \mathrm{C}$ ) and severe metabolic acidosis (VBGA, pH, 6.84; $\mathrm{PCO}_{2}, 25 \mathrm{kPa}$; bicarbonate, $1.6 \mathrm{mmol} / \mathrm{L}$; and base excess, $-28.9 \mathrm{mmol} / \mathrm{L})$. Other laboratory findings included white blood cell count of $15.1 \times 10^{3} / \mu \mathrm{L}(35.0 \%$ neutrophils, $54.0 \%$ lymphocytes, and $11.0 \%$ monocytes), and he moglobin, C-reactive protein, aspartate aminotransferase, ala nine aminotransferase, total bilirubin, direct bilirubin, creatine kinase-MB, troponin-T, troponin-I, N-terminal pro-B type natriuretic peptide, and ammonia levels of $14.4 \mathrm{~g} / \mathrm{dL}, 0.79 \mathrm{mg} / \mathrm{dL}$ (reference, <0.5 mg/dL), $259 \mathrm{U} / \mathrm{L}, 193 \mathrm{U} / \mathrm{L}, 3.5 \mathrm{mg} / \mathrm{dL}, 1.1 \mathrm{mg} /$ $\mathrm{dL}, 27.7 \mathrm{ng} / \mathrm{mL}$ (reference range, 0.1 to $5.8 \mathrm{ng} / \mathrm{mL}$ ), $0.208 \mathrm{ng} /$ $\mathrm{mL}$ (reference range, $<0.1 \mathrm{ng} / \mathrm{mL}$ ), $0.52 \mathrm{ng} / \mathrm{mL}$ (reference range, $<0.16 \mathrm{ng} / \mathrm{mL}$ ), $45,351.0 \mathrm{pg} / \mathrm{mL}$ (reference range, $<84 \mathrm{pg} / \mathrm{mL}$ ), and $323 \mu \mathrm{g} / \mathrm{dL}$, respectively. Moreover, disseminated intravascular coagulation (DIC) was identified (platelet count $58 \times 10^{3} / \mu \mathrm{L}$; prothrombin time international normalized ratio, 4.96; activated partial thromboplastin time, 56.2 seconds; fibrinogen $<50 \mathrm{mg} /$ $\mathrm{dL}$; figrin (ogen) degradation product, $116.2 \mu \mathrm{g} / \mathrm{mL}$; D-dimer, $76.6 \mathrm{mg} / \mathrm{L}$ ). She was admitted to the neonatal intensive care unit for further evaluation. Despite additional fluid resuscitation with high-dose dextrose infusion and oxygen administration via a high-flow nasal cannula, hypoxemia persisted and tachycardia worsened shortly after admission. We immediately performed endotracheal intubation and applied mechanical ventilation. Tachycardia was observed (HR of $240 \mathrm{bpm}$ ) on an ECG monitor. A diagnosis of SVT was made, and adenosine was administered immediately. Afterward, her ECG showed a normal sinus rhythm with an HR of $170 \mathrm{bpm}$, and her dyspnea and lethargy improved. After she became stable, 12-lead ECG was performed, which revealed normal sinus rhythm without ventricular pre-excitation (Figure 2A). Echocardiography showed mild left ventricular dysfunction with mild mitral and tricuspid regurgitation. After she was stabilized, metabolic acidosis gradually improved while maintaining high-dose dextrose and high caloric feeding until completely excluded.

We needed to find possible causes of sudden collapse and severe metabolic acidosis. An inborn error of metabolism was suspected because she had hypoketotic hypoglycemia, hyperam monemia, mildly elevated liver transaminases, and severe meta bolic acidosis. However, newborn screening by tandem mass spectrometry and levels of plasma amino acid, urine organic acid, plasma acylcarnitine, and total and free carnitine suggested that a fatty acid oxidation disorder was unlikely. Furthermore, sepsis was considered because of the systemic inflammatory response showing hypothermia, tachypnea, tachycardia, metabolic acidosis, and DIC. However, there was no obvious increase in the level of

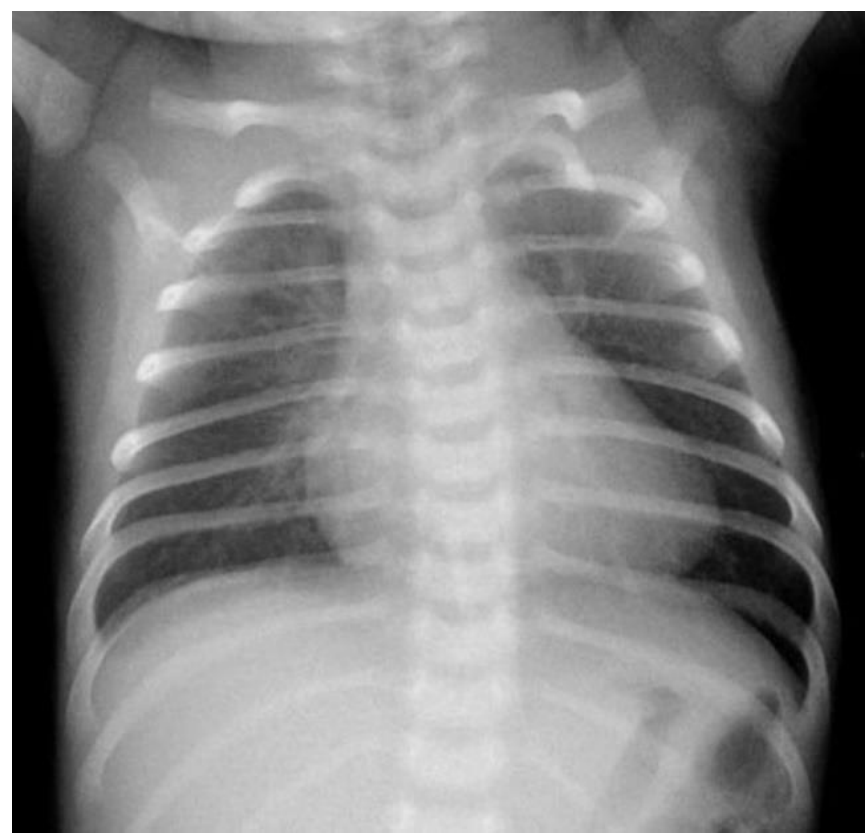

Figure 1. Chest radiograph of a 28-day-old female with cardio vascular collapse shows mild cardiomegaly (cardiothoracic ratio $56 \%$ ) without pulmonary edema. 
inflammatory markers. Additionally, no organism was detected in blood culture, urine culture, and multiplex polymerase chain reaction performed on a nasopharyngeal specimen. Thus, cere brospinal fluid testing was not performed.

On hospitalization day 8, she suddenly developed tachycardia and desaturation. The ECG monitor revealed SVT. Adenosine was administered immediately, and ECG tracing returned to normal sinus rhythm. A subsequent 12-lead ECG showed ventricular pre-excitation diagnostic of WPW syndrome (Figure 2B). Propranolol was started as maintenance treatment of SVT with WPW syndrome. Subsequently, she was discharged on hospitali- zation day 25 without any further events.

A novel sequence variant, c.238A $>\mathrm{G}$ (p.Thr80Ala), in the myosin light chain 2 (MYL2) gene that is related to hypertrophic cardiomyopathy (HCM) and conduction defects on a nextgeneration sequencing-based panel test was found after discharge. Additional parental genetic tests confirmed that the father had the same mutation, but he did not show cardiac symptoms. Further evaluation will be performed on him. The patient is now 16 months old and still receiving beta-blocker treatment in the outpatient clinic. Ventricular pre-excitation remains evident in the follow-up ECG; however, echocardiography revealed normalized

A

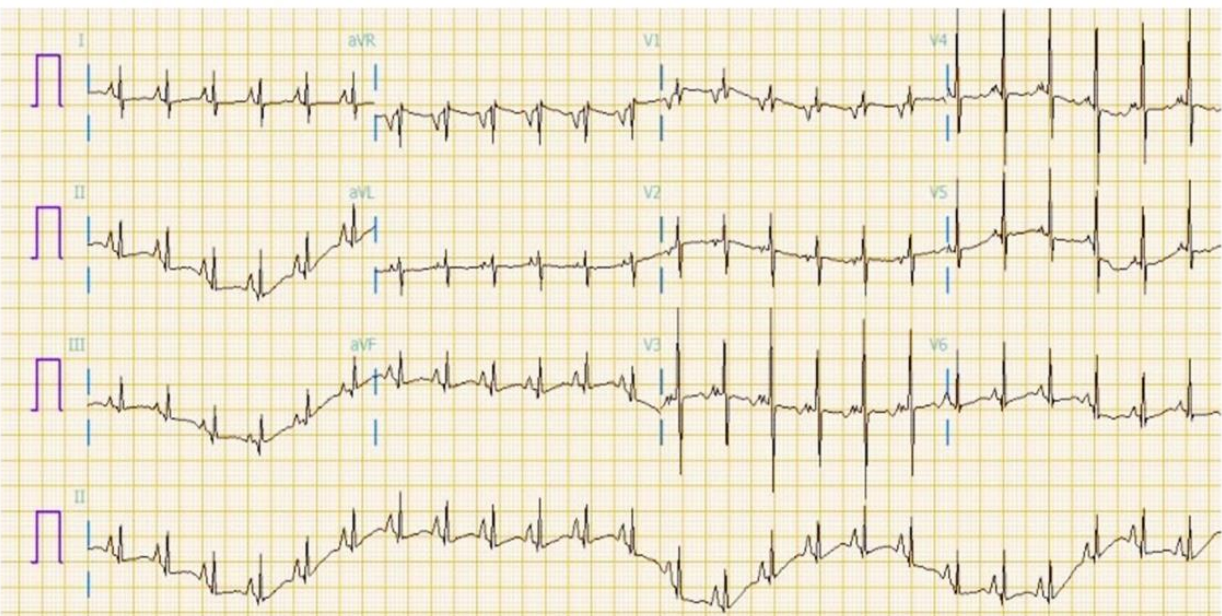

$$
\text { Limb : } 10.00 \mathrm{~mm} / \mathrm{mV} \quad \text { Chest : } 10.00 \mathrm{~mm} / \mathrm{mV} \quad \text { Speed : } 25 \mathrm{~mm} / \mathrm{sec} \quad \mathrm{F} 60 \sim 16.150 \mathrm{~Hz} \text { W Hz }
$$

$\begin{array}{ll}P & 64 \\ R & 76\end{array}$

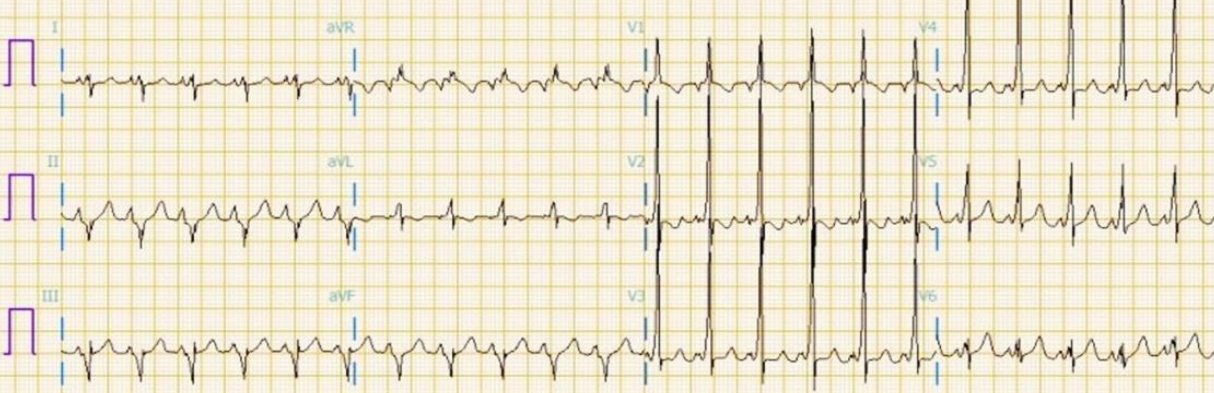

B

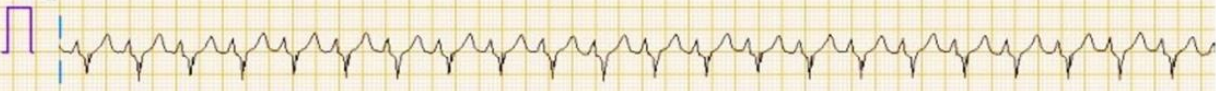

Limb : $10.00 \mathrm{~mm} / \mathrm{mv}$

Chest : $10.00 \mathrm{~mm} / \mathrm{mV}$

Speed : $25 \mathrm{~mm} / \mathrm{sec}$

$\mathrm{F} 60 \sim 16-150 \mathrm{~Hz} W \mathrm{~Hz}$

Figure 2. (A) Electrocardiography tracing of a 28-day-old female with cardiovascular collapse. Electrocardiography after the first episode of supraventricular tachycardia shows normal sinus rhythm (heart rate of 150 beats per minute). (B) Electrocardiography after the second episode of supraventricular tachycardia shows a short PR interval and wide QRS complex in all leads. This indicates ventricular pre-excitation (delta wave) resulting in the diagnosis of Wolff-ParkinsonWhite syndrome. 
findings.

\section{DISCUSSION}

Hypovolemia, bacterial sepsis, and congenital heart disease are common causes of acute cardiovascular collapse in young infants. SVT is the most common tachyarrhythmia in the pediatric population. In addition, WPW syndrome is rare, with an inci dence rate of $0.1 \%$ to $0.2 \%$ among neonates; however, the first manifestation may be shock, requiring immediate attention ${ }^{1,2)}$. Furthermore, 50\% of young patients with WPW syndrome with cardiac arrest had no previous cardiac symptoms ${ }^{1,2)}$. Depending on the duration of SVT, symptoms can range from asymptomatic presentation to cardiogenic shock. Poor feeding, lethargy, and irritability may be present with prolonged tachyarrhythmia. These symptoms appear non-specific. Therefore, it can be mis taken for anemia, fever, sepsis, dehydration, and congenital structural cardiac defects ${ }^{3}$. In severe cases, metabolic acidosis, hypoglycemia, hypothermia, poor perfusion, shock, and even cardiac arrest may occur ${ }^{2}$. Furthermore, SVT in neonates is relatively rare; therefore, the initial manifestation is often heart failure ${ }^{4)}$. A review of the literature revealed three reports of six infants total who exhibited cardiogenic shock from prolonged SVT with normal HR and sinus rhythm on admission ${ }^{4-6)}$. Similar to other reports, our patient had normal HR and sinus rhythm initially, and delta waves were not detected in the first $\mathrm{ECG}^{7,8)}$. Therefore, arrhythmia should be considered, and both ECG and vital signs monitoring should be conducted in critically ill patients. In addition, serial 12-lead ECG is necessary for all patients with arrhythmia.

Early diagnosis is crucial for WPW syndrome because this can lower morbidity and mortality. In neonates, arrhythmia is con sidered benign. However, if the HR is consistently $>180 \mathrm{bpm}$ at rest, there may be hemodynamic instability; hence, ECG should be performed immediately ${ }^{1)}$. ECG is the gold standard for the diagnosis of WPW syndrome. WPW syndrome can be diagnosed by a short PR interval ( $<0.12$ seconds), prolonged QRS duration ( $>0.08$ seconds), and delta wave. ECG should be performed for the detection of SVT. In addition, if adenosine is administered after an SVT episode to suppress atrioventricular node conduction, the possibility of finding ventricular pre-excitation through a delta wave increases ${ }^{9)}$.

Usually, WPW syndrome occurs sporadically and is due to a disorder during embryonic development. However, $3.4 \%$ of cases who had first-degree relatives with pre-excitation syndrome are diagnosed as WPW syndrome, which is a known risk factor of WPW syndrome. The familial inheritance pattern is autosomaldominant and related genetic variations are known ${ }^{10}$. Chromosome 20 abnormalities induce HCM with WPW syndrome, and mutations of the protein kinase AMP-activated non-catalytic subunit gamma 2 (PRKAG2) gene on chromosome 7 induces cardiac glycogen overload ${ }^{11)}$. Genetic analysis is necessary for understanding the inheritance patterns of familial WPW syndrome and future gene therapy.

Congenital or acquired heart disease is present in $20 \%$ of children with WPW syndrome. Up to $37 \%$ of infants diagnosed with WPW syndrome may have congenital heart malformations, such as Ebstein anomaly, congenitally corrected transposition of the great arteries, and ventricular septal defects. In 18\%, the patients with cardiac rhabdomyomas associated with tuberous sclerosis are diagnosed as WPW syndrome ${ }^{1}$. Congenital heart disease can cause severe hypotension or cardiac arrest during SVT. Echocardiography is limited in the diagnosis of WPW syndrome; however, it provides information about associated heart disease while providing a view of the structure and function of the heart.

Children with SVT diagnosed in the neonatal period are advised to discontinue the drug by 12 months of age. Gilljam et al. ${ }^{12)}$ reported that most neonates received antiarrhythmic drug treatment for 6 to 12 months and did not have SVT recurrences. By contrast, SVT associated with ventricular pre-excitation is more likely to persist and requires long-term antiarrhythmic treatment as shown by Riggs et al. ${ }^{13)}$. Therefore, in patients in whom arrhythmia is initially difficult to treat and/or those who have ventricular pre-excitation during sinus rhythm, continuation of prophylactic drugs for a longer period was previously con sidered.

The MYL2 gene encodes the regulator light chain associated with cardiac myosin beta heavy chain. Calcium triggers phosphorylation of the regulatory light chain, which in turn, triggers contraction. Mutations in this gene are associated with conduction defects and mid-left ventricular chamber-type $\mathrm{HCM}^{14)}$. Andersen et al. ${ }^{15)}$ reported that more than 50 genes have been associated with $\mathrm{HCM}$, and clinical manifestations can range from asymptomatic to severe disease at an early age. Claes et al. ${ }^{16)}$ reported that patients who were MYL2 mutation carriers showed a high likelihood of HCM related to hypertension. Missense mutations are the main mutation type found in the MYL2 gene. In diagnostic testing datasets, the c.238A $>\mathrm{G}$ (p.Thr80Ala) mutation of the MYL2 gene is a novel variant that has not been reported in the 
normal population databases (gnomAD, Korean Reference Genome DB [KRGDB]). In silico prediction (SIFT, PolyPhen-2, MutationTaster) predicted that the mutation is deleterious. More over, the p.Thr80Ala mutation detected in the present case has an identical amino acid codon position as the p.Thr80Asn mutation, which is a known HCM-related pathogenic mutation ${ }^{14)}$. This can serve as further evidence suggesting pathogenicity even if amino acid changes are not the same. Ho et al. ${ }^{17)}$ reported that a 5-year follow-up of asymptomatic patients with a p.Thr80Asn mutation showed the presence of altered cardiac dimensions in all patients. In our case, no structural abnormality was found on echocardio graphy. To determine the development of abnormal findings, re gular and routine follow-ups are indicated along with the medical evaluation of family members.

In conclusion, this case shows that a neonate with WPW syndrome, presenting with cardiogenic shock and mimicking inborn error of metabolism, can be successfully diagnosed and treated with beta-blockers after suspecting arrhythmia and performing subsequent ECG. Cardiogenic shock should be considered, despite being a rare cause of shock among neonates. Moreover, to determine whether the c.238A $>\mathrm{G}$ (p.Thr80Ala) mutation of the MYL2 gene is a pathogenic mutation related to HCM and con duction defects, long-term follow-up and studies are required.

\section{ARTICLE INFORMATION}

\section{Ethical statement}

This retrospective chart review study involving human parti cipants was in accordance with the ethical standards of the institutional and national research committee and with the 1964 Helsinki Declaration and its later amendments or comparable ethical standards. This study was approved by the Institutional Review Board of Soonchunhyang University Bucheon Hospital (SCHBC 2020-04-001). Written informed consent by the patient was waived due to a retrospective nature of our study.

\section{Conflicts of interest}

No potential conflict of interest relevant to this article was reported.

\section{Author contributions}

Conception or design: S.H.L., G.Y.P., Y.L.S., S.S.K.

Acquisition, analysis, or interpretation of data: J.E.H, S.H.L.
Drafting the work or revising: J.E.H, S.H.L., M.A.J.

Final approval of the manuscript: J.E.H, S.H.L., G.Y.P., Y.L.S., S.S.K., M.A.J.

\section{ORCID}

Ji Eun Ha https://orcid.org/0000-0001-6614-2683

Sun Hyang Lee https://orcid.org/0000-0001-7873-8409

\section{Acknowledgments}

None

\section{REFERENCES}

1. Chambers S, Jnah A, Newberry D. The pathophysiology, diag nosis, and management of Wolff-Parkinson-White syndrome in the neonate. Adv Neonatal Care 2020 Aug 19 [Epub]. https:// doi.org/10.1097/ANC.0000000000000785.

2. Hoeffler CD, Krenek ME, Brand MC. Wolff-Parkinson-White syndrome in a term infant presenting with cardiopulmonary arrest. Adv Neonatal Care 2016;16:44-51.

3. Tsao S, Deal BJ. Management of symptomatic Wolff-ParkinsonWhite syndrome in childhood. Prog Pediatr Cardiol 2013;35:715.

4. Dharnidharka VR, Lieh-Lai M, Sarnaik A, Clapp S. A child with cardiogenic shock and supraventricular tachycardia presenting in normal sinus rhythm. Pediatr Emerg Care 1996;12:420-1.

5. Gikonyo BM, Dunnigan A, Benson DW Jr. Cardiovascular collapse in infants: association with paroxysmal atrial tachycardia. Pediatrics 1985;76:922-6.

6. Viveiros E, Aveiro AC, Costa E, Nunes JL. Cardiogenic shock in a neonate. BMJ Case Rep 2013;2013:bcr2012008440.

7. Al-Khatib SM, Pritchett EL. Clinical features of Wolff-ParkinsonWhite syndrome. Am Heart J 1999;138(3 Pt 1):403-13.

8. Munger TM, Packer DL, Hammill SC, Feldman BJ, Bailey KR, Ballard DJ, et al. A population study of the natural history of Wolff-Parkinson-White syndrome in Olmsted County, Minnesota, 1953-1989. Circulation 1993;87:866-73.

9. Richardson C, Silver ES. Management of supraventricular tachycardia in infants. Paediatr Drugs 2017;19:539-51.

10. MacRae CA, Ghaisas N, Kass S, Donnelly S, Basson CT, Watkins HC, et al. Familial hypertrophic cardiomyopathy with WolffParkinson-White syndrome maps to a locus on chromosome 7q3. J Clin Invest 1995;96:1216-20.

11. van der Steld LP, Campuzano O, Perez-Serra A, Moura de Barros Zamorano M, Sousa Matos S, Brugada R. Wolff-Parkinson-White syndrome with ventricular hypertrophy in a Brazilian family. Am J Case Rep 2017;18:766-76. 
12. Gilljam T, Jaeggi E, Gow RM. Neonatal supraventricular tachycardia: outcomes over a 27-year period at a single institution. Acta Paediatr 2008;97:1035-9.

13. Riggs TW, Byrd JA, Weinhouse E. Recurrence risk of supraventricular tachycardia in pediatric patients. Cardiology 1999;91:2530.

14. National Center for Biotechnology Information, U.S. National Library of Medicine. MYL2 myosin light chain 2 [homo sapiens (human)] [Internet]. Bethesda: NCBI Gene; 2020 [cited 2021 May 12]. Available from: http://www.ncbi.nlm.nih.gov/gene/ 4633.

15. Andersen PS, Havndrup O, Hougs L, Sorensen KM, Jensen M,
Larsen LA, et al. Diagnostic yield, interpretation, and clinical utility of mutation screening of sarcomere encoding genes in Danish hypertrophic cardiomyopathy patients and relatives. Hum Mutat 2009;30:363-70.

16. Claes GR, van Tienen FH, Lindsey P, Krapels IP, Helderman-van den Enden AT, Hoos MB, et al. Hypertrophic remodelling in cardiac regulatory myosin light chain (MYL2) founder mutation carriers. Eur Heart J 2016;37:1815-22.

17. Ho CY, Day SM, Colan SD, Russe 11 MW, Towbin JA, Sherrid MV, et al. The burden of early phenotypes and the influence of wall thickness in hypertrophic cardiomyopathy mutation carriers: findings from the HCMNet study. JAMA Cardiol 2017;2:419-28. 